\title{
Ubiquitin specific peptidase 19 is a prognostic biomarker and affect the proliferation and migration of clear cell renal cell carcinoma
}

\author{
WENBING HU ${ }^{1 *}$, YANFANG SU $^{2 *}$, XINXIONG FEI $^{1}$, XUDONG WANG $^{1}$, GUANGLIN ZHANG $^{1}$, \\ CHUNYAN SU ${ }^{1}$, TIANXING DU ${ }^{1}$, TAO YANG ${ }^{1}$, GANGSHENG WANG $^{1}$, ZE TANG $^{1}$ and JIERONG ZHANG ${ }^{1}$ \\ ${ }^{1}$ Department of Oncology, Huangshi Central Hospital of Edong Healthcare, \\ Affiliated Hospital of Hubei Polytechnic University, Huangshi, Hubei 435000; \\ ${ }^{2}$ Medical Science Research Center, Zhongnan Hospital of Wuhan University, Wuhan, Hubei 430000, P.R. China
}

Received June 15, 2019; Accepted February 14, 2020

DOI: $10.3892 /$ or.2020.7565

\begin{abstract}
Ubiquitin specific peptidase 19 (USP19) is a member of the USP family and exhibits diverse roles in various biological processes, such as cell differentiation, cell cycle progression and apoptosis. There is limited knowledge regarding the role and impact of USP19 in cancer, particularly clear cell renal cell carcinoma (ccRCC). To examine the function of USP19 in ccRCC, The Cancer Genome Atlas (TCGA) and Gene Expression Omnibus databases were examined to determine USP19 mRNA expression levels. USP19 mRNA levels were significantly lower in ccRCC tissues than in normal tissues. USP19 downregulation was associated with ccRCC progression and poor prognostic outcomes in TCGA cohort. Furthermore, the functional involvement of USP19 in ccRCC was examined using Cell Counting Kit-8, soft agar, Transwell and wound healing assays in vitro following overexpression or knockdown of USP19 in the Caki-1 cell line. USP19 overexpression inhibited ccRCC proliferation and migration, whereas USP19 knockdown promoted ccRCC proliferation and migration in vitro. Consistent with these results, it was further demonstrated that USP19 downregulation promoted tumor growth in vivo in a xenograft model. Mechanistically, it was found that USP19 exerted its inhibitory effect on ccRCC proliferation and migration by suppressing the activation of ERK. Collectively, the present findings identified a role for
\end{abstract}

Correspondence to: Dr Ze Tang or Dr Jierong Zhang, Department of Oncology, Huangshi Central Hospital of Edong Healthcare, Affiliated Hospital of Hubei Polytechnic University, 293 Hospital Avenue, Xisaishan, Huangshi, Hubei 435000, P.R. China

E-mail: 1263326939@qq.com

E-mail: 490935066@qq.com

${ }^{*}$ Contributed equally

Key words: ubiquitin specific peptidase 19, clear cell renal cell carcinoma, suppressor, proliferation, migration
USP19 as a tumor suppressor in ccRCC and demonstrated that USP19 is a potential prognostic biomarker that could be applied in ccRCC therapy.

\section{Introduction}

Renal cell carcinoma (RCC) is one of the most prevalent malignancies of the urinary system, accounting for $2.2 \%$ of all adult malignancies globally, with estimations of 403,262 new cases and 175,098 deaths worldwide in 2018 (1). Clear cell (cc)RCC represents the predominant histologic subtype of RCC and constitutes $\sim 70 \%$ of all cases (2). The remaining $30 \%$ of RCC subtypes, such as chromophobe and papillary RCC, are generally indolent (3). Surgery is the most effective treatment for ccRCC, as chemotherapy and radiotherapy are not as successful in controlling its progression; however, 25\% of clinical patients with ccRCC will develop metastatic disease despite curative surgical removal of the primary tumor (4). The overall 5-year survival rate of RCC patient is $5-10 \%$, with a median survival of only $\sim 13$ months (5). Therefore, the molecular mechanisms underlying ccRCC initiation and progression require improved understanding, which may contribute to the development of novel strategies for treating ccRCC.

Ubiquitin and ubiquitin-like modifications participate in most cellular signaling pathways (6). Ubiquitination is reversed by deubiquitinating enzymes (DUBs) that cleave ubiquitin from the substrate protein (7). There are $\sim 98$ DUBs known to be encoded by the human genome, which are grouped into six subfamilies, the largest one being the ubiquitin specific peptidase (USP) family (8). The USP family exhibits a conserved three-domain architecture, comprising the palm, thumb and fingers of a right hand (9). USP19 belongs to the USP family and several studies have reported that USP19 exhibits diverse roles in biological processes. For example, USP19 is involved in the endoplasmic reticulum-associated degradation pathway and the unfolded protein response (10). Additionally, USP19 has also been identified as a positive regulator involved in regulating autophagy and innate immune responses (11). Furthermore, USP19 also reported to have involved in the pathogenesis of certain cancers. For example, Ewing 
sarcoma breakpoint region 1-Friend leukemia integration 1 (EWS-FLI1) is a chimeric oncoprotein uniquely expressed in tumor cells, and constant EWS-FLI1 protein turnover is regulated by the ubiquitin proteasome system (12). USP19 as a specific modulator of EWS-FLI1 protein stability, which mediates an increase in fusion protein deubiquitination by binding to its $\mathrm{N}$-terminal domain to regulate Ewing sarcoma growth (12). In addition, BRCA1-associated protein 1 (BAP1) is a tumor suppressor gene important to the development and prognosis of numerous cancers (13); Shahriyari et al (13) found that USP19 was highly positively associated with BAP1 expression in breast and uveal melanoma. At present, there is limited knowledge regarding the role and impact of USP19 in other cancers, including ccRCC.

In the present study, the expression and clinical significance of USP19 were explored in The Cancer Genome Atlas (TCGA) and Gene Expression Omnibus (GEO) ccRCC samples, and the biological functions of USP19 in ccRCC were investigated in vitro and in vivo.

\section{Materials and methods}

Date sources. Level 3 mRNA sequencing (seq) data (downloaded in November 2016) of ccRCC, also known as kidney renal clear cell carcinoma (KIRC), including 539 tumor samples and 72 adjacent normal samples, were downloaded from TCGA database (KIRC RNA-seq; http://www.cbioportal.org/). Clinical information, including age, sex, histological grade, tumor stage, survival time and outcome, were also extracted from TCGA. The copy numbers of 587 ccRCC tissues and 901 paired normal kidney tissues from the TCGA database (KIRC CNV) were obtained. GSE76207 (14) and GSE102101 (15) were obtained from the GEO database (http://www.ncbi.nlm. nih.gov/geo/), and respectively contained 16 and 10 paired tumor and normal kidney tissues.

Bioinformatics analysis of the USP19 transcriptional levels in $c c R C C$. The KIRC RNA-seq level 3 count data and GSE76207 data were normalized with the negative binomial distribution methodology by R (version 3.4.1) (16) package DESeq2 (17). The GSE102101 sequence reads were mapped to the human genome (hg38) using HISAT2 (version 2.1.0; http://ccb.jhu. edu/software/hisat $2 /$ ) and transcripts were quantified using stringtie (version 1.3.4; http://ccb.jhu.edu/software/stringtie/) with refseq annotation, normalized and subjected to differential expression analysis using DESeq2.

Copy number variation analysis. The KIRC copy-number variation (CNV) data were annotated by bedtools (https://bedtools. readthedocs.io/).

Subgroup analysis based on clinicopathologic features. To further explore the role of USP19, KIRC tumor samples were divided into various groups based on their clinicopathological features (age, sex, histological grade and stage). Then, differential expression analysis of the tumor samples in each group was conducted by t-test.

Survival analysis. Gene Expression Profiling Interactive Analysis (http:/gepia.cancer-pku.cn/) was used to analyze the overall survival (OS) and disease-free survival (DFS) differences between patients with high or low expression levels of USP19. Patients above the upper quartile with respect to USP19 expression were classified as having a high expression levels, whereas those below the lower quartile were classified as having a low expression level. Those patients with USP19 expression values between the lower and upper quartiles were excluded from survival analysis. Kaplan-Meier analysis and a log-rank test was used to calculate the significance of survival time differences between the two classes of patients.

Plasmid construction and transfection. Full-length human USP19 mRNA was cloned from a human cDNA library and inserted into the mammalian expression vector $\mathrm{pHAGE}-3 \mathrm{x}$ flag (Invitrogen; Thermo Fisher Scientific, Inc.) to construct a pHAGE-3xflag-USP19 plasmid. Two short hairpin RNAs (shRNAs) targeting different regions of USP19 mRNA (shUSP19\#1 and shUSP19\#2) were inserted into the pLKO.1 vector (OligoEngine) and confirmed by sequencing. The sequences of the shRNAs were 5'-CTCCACTGCGAGCGA AGTATT-3' (shUSP19\#1), 5'-CCGGTACTCTGTGAGTGT ATT-3' (shUSP19\#2) and 5'-GUCAGCGUGCAGAUAGAG UUU-3' [control shRNA (shctrl)]. Lentiviral supernatants were harvested $48 \mathrm{~h}$ after $293 \mathrm{~T}$ cells at $80-90 \%$ confluency were co-transfected with packing plasmid $(0.5 \mu \mathrm{g}$ pMD2.G and $0.75 \mu \mathrm{g}$ psPAX2; Novagen; Merck KGaA) and $1 \mu \mathrm{g}$ pHAGE-3xflag-USP19, empty pHAGE-3xflag, shUSP19\#1, shUSP19\#2 or shctrl plasmid using polyetherimide transfection reagent (cat. no. GF95977287; Sigma-Aldrich; Merck $\mathrm{KGaA}$ ). The lentivirus was harvested $48 \mathrm{~h}$ after transfection, and Caki- 1 cells $\left(5 \times 10^{5}\right.$ cells/well) were incubated with lentivirus-containing medium (MOI=50) supplemented with $2 \mu \mathrm{l}$ polybrene (Sigma-Aldrich; Merck KGaA). At 48 h later, stable Caki-1 cells were selected in the presence of $4 \mu \mathrm{g} / \mathrm{ml}$ puromycin (Sigma-Aldrich; Merck KGaA) for 2 days. USP19 mRNA expression was examined by reverse transcription-quantitative PCR (RT-qPCR) and protein expression was evaluated using western blotting.

Cell lines and reagents. 293T cells were obtained from American Type Culture Collection. The human renal cancer cell line Caki-1 was purchased from the Cell Bank of the Type Culture Collection of the Chinese Academy of Sciences. 293T and Caki-1 cells were cultured in DMEM (cat. no. C11995500BT; Gibco; Thermo Fisher Scientific, Inc.) supplemented with 10\% FBS (cat. no. F04-001; Biological Industries) and 1\% penicillin/streptomycin (cat. no. 15140-122; Gibco; Thermo Fisher Scientific, Inc.), and the cells were cultured in a humidified atmosphere containing $5 \% \mathrm{CO}_{2}$ at $37^{\circ} \mathrm{C}$. U0126 (cat. no. HY-12031; MedChemExpress LLC), an ERK inhibitor, was dissolved in DMSO and used to treat Caki-1 cells at a concentration of $20 \mu \mathrm{m}$.

Cell proliferation. Cell Counting Kit-8 (CCK-8) assays were performed to evaluate the proliferation capacity of Caki-1 cells. Cells were seeded into 96 -well plates at a density of $3 \times 10^{3} /$ well. After incubation for various timepoints $(0,24,48,72$ and $96 \mathrm{~h})$, $10 \mu$ l CCK-8 reagent (Dojindo Molecular Technologies, Inc.) was added to each well, followed by incubation for a further $4 \mathrm{~h}$ at room temperature. Then, the cell proliferation ability 
Table I. Primer sequences.

\begin{tabular}{ll}
\hline Gene & \multicolumn{1}{c}{ Primer } \\
\hline p27 & F: 5'-GGTGGAGAAGGGCAGCTTG-3' \\
& R: 5'-GAAGAATCGTCGGTTGCAGG-3' \\
PCNA & F: 5'-CACTCCACTCTCTTCAACGGT-3' \\
& R: 5'-ATCCTCGATCTTGGGAGCCA-3' \\
Cyclin D1 & F: 5'-CAGATCATCCGCAAACACGC-3' \\
& R: 5'-AGGCGGTAGTAGGACAGGAA-3' \\
MMP2 & F: 5'-CCGTCGCCCATCATCAAGTT-3' \\
& R: 5'-CCGCATGGTCTCGATGGTAT-3' \\
MMP9 & F: 5'-TTTGAGTCCGGTGGACGATG-3' \\
& R: 5'-TTGTCGGCGATAAGGAAGGG-3' \\
USP19 & F: 5'-TAAATCCAAGGCACGATCTGAGG-3' \\
& R: 5'-GCTTTGGGGTTACATGCTCCA-3' \\
18S rRNA & F: 5'-GTAACCCGTTGAACCCCATT-3' \\
& R: 5'-CCATCCAATCGGTAGTAGCG-3'
\end{tabular}

PCNA, proliferating cell nuclear antigen; MMP, matrix metalloproteinase; USP19, ubiquitin specific peptidase 19; rRNA, ribosomal RNA.

was examined by measuring the absorbance at $450 \mathrm{~nm}$ using an enzyme label analyzer.

For soft agar assays, 3,000 cells were seeded onto 6-well plates containing $0.5 \%$ top agarose and $1 \%$ bottom agarose, and incubated for 2 weeks in a humidified atmosphere containing $5 \% \mathrm{CO}_{2}$ at $37^{\circ} \mathrm{C}$. The number of colonies in each plate was counted using Image $\mathrm{J}$ software version 1.47 (National Institutes of Health).

Transwell migration assay. Migration assays were performed using $5-\mathrm{mm}$ Transwell ${ }^{\circledR}$ inserts with $5-\mu \mathrm{m}$ pore polycarbonate membranes (Corning, Inc.). A $0.5-\mathrm{ml}$ cell suspension $\left(2.5 \times 10^{4}\right.$ cells $)$ in serum-free culture medium was added to the upper chambers, and $750 \mu \mathrm{l}$ culture medium containing $10 \%$ FBS was added to the lower chambers. After $24 \mathrm{~h}$, the cells on the upper surface of the membrane were quickly wiped away with cotton-tipped swabs, and the cells on the lower surface were fixed with $4 \%$ paraformaldehyde for $15 \mathrm{~min}$ at room temperature, following by staining with a $0.1 \%$ crystal violet solution (Sigma-Aldrich; Merck KGaA) for $30 \mathrm{~min}$ at room temperature. Cell counting was performed by photographing the membrane through a light microscope. Four random microscopic fields (magnification, x100) in each chamber were photographed.

Wound healing assay. Cells were seeded at $5 \times 10^{5}$ cells/well into 6-well plates. After the cells reached $\sim 90 \%$ confluence, three separate wounds were scratched through the cells with a sterile $100-\mu 1$ pipette tip. Floating cells were removed with PBS and the medium was replaced with serum-free medium. Images were captured at 0 and $48 \mathrm{~h}$ following the initial scratch to evaluate cell migration. Cell migration rate $(\%)=[$ (scratch width at $0 \mathrm{~h}$-scratch width at $48 \mathrm{~h}$ )/original scratch width $\mathrm{x}$ $100 \%]$.
Table II. Relationship between the expression of USP19 and clinicopathological characteristics.

\begin{tabular}{lccc}
\hline Characteristic & Cases, $\mathrm{n}$ & $\begin{array}{c}\text { USP19 mean } \\
\text { expression value }\end{array}$ & P-value \\
\hline Age & & & 0.2254 \\
$\quad \leq 60$ & 249 & $2,749.977 \pm 913.719$ & \\
$>60$ & 289 & $2,661.380 \pm 755.453$ & \\
Sex & & & 0.0933 \\
$\quad$ Male & 353 & $2,658.620 \pm 827.082$ & \\
Female & 186 & $2,785.718 \pm 837.334$ & \\
Histological grade & & & 0.0086 \\
$\quad$ Low & 249 & $2,775.497 \pm 890.832$ & \\
High & 282 & $2,592.384 \pm 677.281$ & \\
Stage & & & $<0.0001$ \\
I-II & 331 & $2,832.380 \pm 961.756$ & \\
III-IV & 205 & $2,496.370 \pm 507.991$ & \\
\hline
\end{tabular}

USP, ubiquitin specific peptidase 19.

RNA extraction and RT-qPCR. Total RNA from cells were extracted using TRIzol ${ }^{\circledR}$ reagent (Invitrogen; Thermo Fisher Scientific, Inc.) according to the manufacturer's protocols. For qPCR assays, total RNA was reverse transcribed into cDNA using a Transcriptor First Strand cDNA Synthesis Kit (Roche Diagnostics) according to the manufacturer's protocols. The expression of USP19 was measured by qPCR using SYBR Green PCR Master Mix (Roche Diagnostics). qPCR was performed as follows: Initial denaturation for $2 \mathrm{~min}$ at $55^{\circ} \mathrm{C}$ and $30 \mathrm{sec}$ at $95^{\circ} \mathrm{C}$, followed by 35 cycles of $95^{\circ} \mathrm{C}$ for $5 \mathrm{sec}$ and $60^{\circ} \mathrm{C}$ for $30 \mathrm{sec}$. $18 \mathrm{~S}$ ribosomal RNA was used as an internal control, and the relative mRNA expression was estimated using the $2^{-\Delta \Delta C q}$ method (18). The primer sequences are shown in Table I.

Protein extraction and western blot assay. Total protein was extracted using RIPA lysis buffer (50 mM Tris-HCl, $\mathrm{pH} 7.4$, $150 \mathrm{mM} \mathrm{NaCl}, 1 \%$ Triton X-100, $1 \%$ sodium deoxycholate, $0.1 \%$ SDS and $1 \mathrm{mM}$ EDTA) containing a protease inhibitor cocktail (cat. no. 4963124001; Roche Diagnostics). The protein concentrations were quantified using a bicinchoninic acid assay kit, and 30-50 $\mu$ g protein was boiled for $5 \mathrm{~min}$ and used for western blot analysis. The proteins were separated by $8-12 \%$ SDS-PAGE and transferred to PVDF membranes at $200 \mathrm{~mA}$ for $1 \mathrm{~h}$. The PVDF membranes were blocked with $5 \%$ nonfat milk at room temperature for $1 \mathrm{~h}$. Next, the membranes were incubated with primary antibodies $(1: 1,000)$ at $4{ }^{\circ} \mathrm{C}$ overnight and washed with TBS- $0.1 \%$ Tween-20 three times. The membranes were further incubated with horseradish peroxidase (HRP)-conjugated secondary antibodies (1:10,000; cat. nos. 111-035-003 and 115-035-003; Jackson ImmunoResearch Laboratories, Inc.) at $37^{\circ} \mathrm{C}$ for $1 \mathrm{~h}$. Finally, the membranes were developed by Femto ECL substrates (Thermo Fisher Scientific, Inc.) and captured by a ChemiDoc system (Bio-Rad Laboratories, Inc.) containing a Chemi HR camera. The images were analyzed by Image Lab 

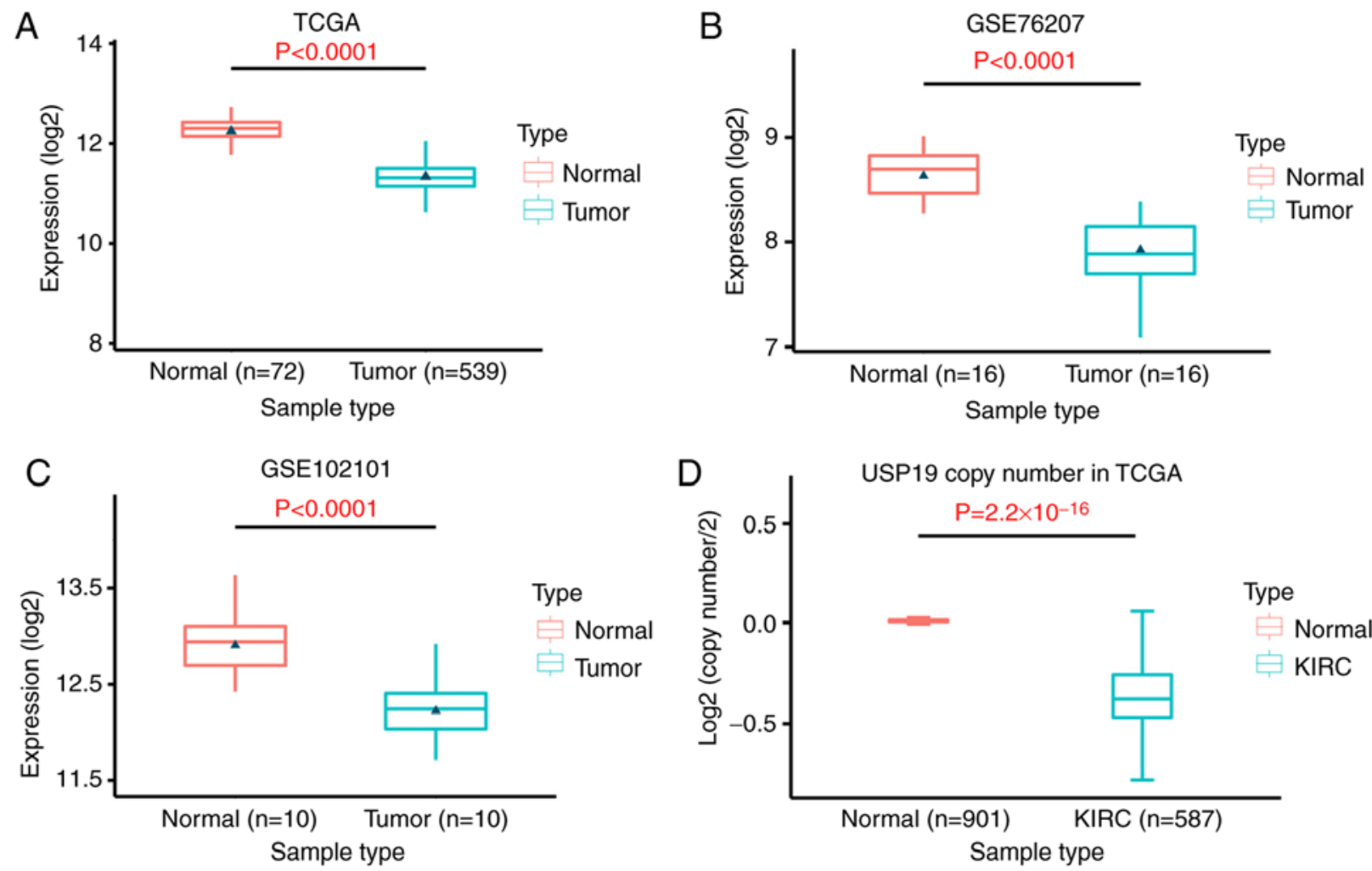

Figure 1. USP19 is downregulated in clear cell renal cell carcinoma tissue. USP19 expression was downregulated in renal cancer tissues compared with in normal renal tissues in (A) TCGA, (B) GSE76207 and (C) GSE102101 datasets. (D) USP19 copy number was significantly lower in KIRC tissues than in normal tissues. TCGA, The Cancer Genome Atlas; KIRC, kidney renal clear cell carcinoma; USP, ubiquitin specific peptidase.

3.1 software (Bio-Rad Laboratories, Inc.). Primary antibodies targeting USP19 (cat. no. ab93159; Abcam), p27 (cat. no. 3688; Cell Signaling Technology, Inc.), proliferating cell nuclear antigen (PCNA; cat. no. 2586; Cell Signaling Technology, Inc.), cyclin D1 (cat. no. 2922; Cell Signaling Technology, Inc.), matrix metalloproteinase (MMP)2 (cat. no. A11144; ABclonal Biotech Co., Ltd.), MMP9 (cat. no. A0289; ABclonal Biotech Co., Ltd.), phosphorylated (p)-ERK (cat. no. 4370; Cell Signaling Technology, Inc.), ERK (cat. no. 4695; Cell Signaling Technology, Inc.), $\beta$-actin (cat. no. 4967; Cell Signaling Technology, Inc.) were used.

Xenograft model. All animal protocols conducted in the present study were approved by the Institutional Animal Care and Use Committee of the Institute of Model Animals of Wuhan University. All animal care outlined in our study were performed in adherence with the Guide for the Care and Use of Laboratory Animals published by US National Institutes of Health (19). The following criteria were set as humane endpoints for the study: Abnormal behavior; $\pm 20 \%$ change in body weight; tumor diameter $>1.5 \mathrm{~cm}$; or tumor ulceration. The maximum tumor diameter was not exceeded until day 60 of the study, when animals were sacrificed; the maximum diameter observed in any one direction was $1.95 \mathrm{~cm}$.

A total of $10 \mathrm{BALB} / \mathrm{c}$ female mice (4-6 weeks; 17-18 g) were purchased from Beijing Vital River Laboratory Animal Technology Co., Ltd. and housed under specific pathogen-free conditions and a 12 -h light/dark cycle, at $25^{\circ} \mathrm{C}$ with $60 \pm 10 \%$ humidity, and free access to food and water. A total of $5 \times 10^{6}$ stably infected cells were diluted in $150 \mu \mathrm{l}$ DMEM and
Matrigel, and inoculated subcutaneously into the left flank of each mouse (5 mice/group). The tumor volumes (V) were measured every 5 days, and calculated using the following formula: $\mathrm{V}=0.5 \mathrm{x}$ (length $\mathrm{x}$ width $^{2}$ ). At 60 days after injection, mice were sacrificed via cervical dislocation. If no spontaneous breathing was observed for $3 \mathrm{~min}$ and the blink reflex was lost, mice were considered to be euthanized. During the study, no animals were prematurely euthanized or found dead. The tumors were removed from the mice, weighed and used for western blot assays.

Statistical analysis. The data were presented as the mean \pm SD of $\geq 3$ experimental repeats using GraphPad Prism 5 software (GraphPad Software, Inc.). Comparisons between two groups were performed using two-tailed Student's t-tests. Differences among 3 groups were assessed using one-way ANOVA followed by Bonferroni test (equal variances assumed) or Tamhane's T2 test (equal variances not assumed). Differences among 4 groups were assessed using two-way ANOVA followed by Bonferroni test. Statistical analyses were performed using SPSS 19 Statistics software (IBM Corp.). $\mathrm{P}<0.05$ was considered to indicate a statistically significant difference.

\section{Results}

USP19 is downregulated due to copy number loss in ccRCC tissues. To explore whether USP19 was involved in the pathogenesis of ccRCC, its expression was first detected in patients with ccRCC and healthy controls. RNA-seq data from the samples of 539 patients with ccRCC from TCGA database 
A

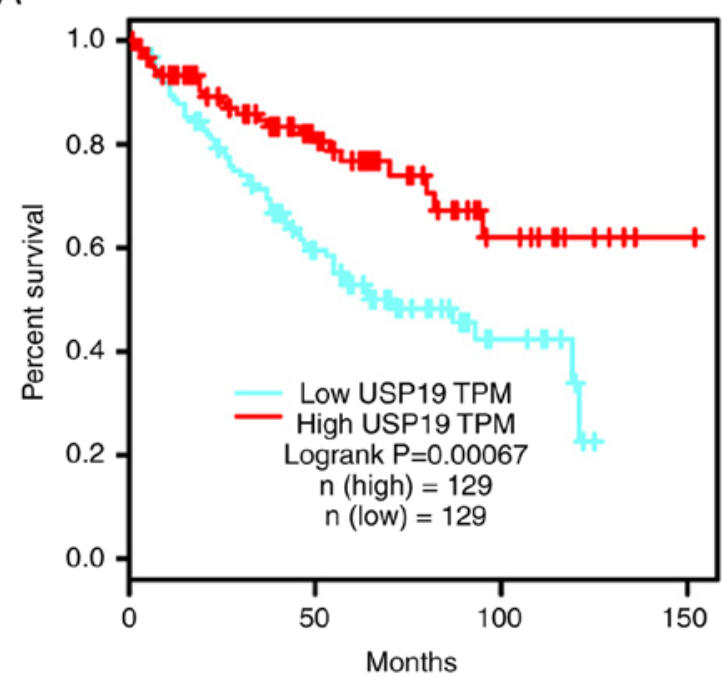

B

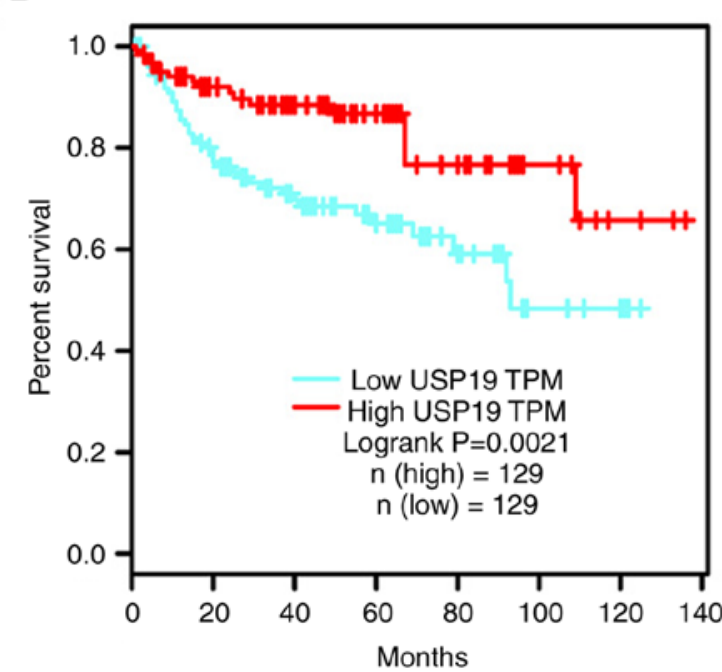

Figure 2. Low USP19 expression is associated with clear cell renal cell carcinoma prognosis. Gene Expression Profiling Interactive Analysis revealed associations between USP19 expression and (A) OS and (B) DFS rates according to The Cancer Genome Atlas kidney renal clear cell carcinoma data. OS, overall survival; DFS, disease-free survival; USP, ubiquitin specific peptidase; TPM, transcript per million.

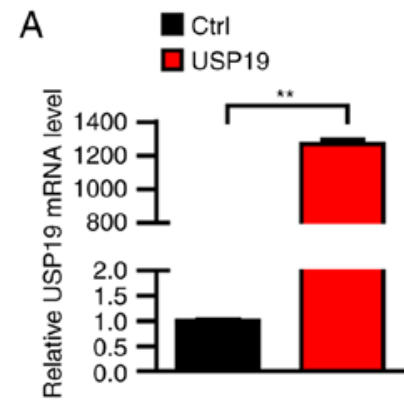

$\mathrm{D}$

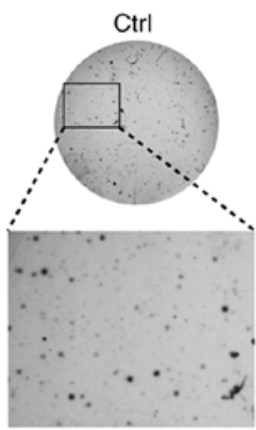

$\mathrm{E}$

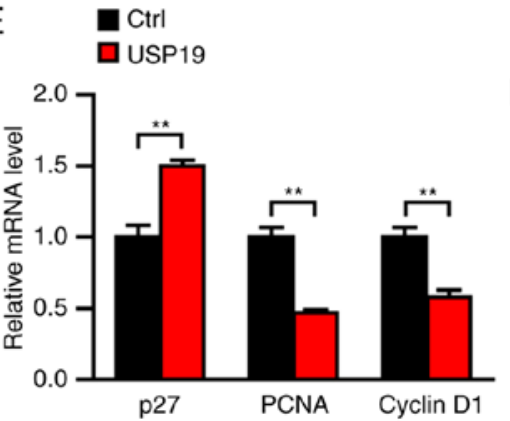

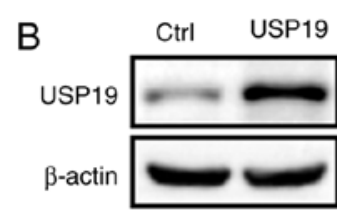

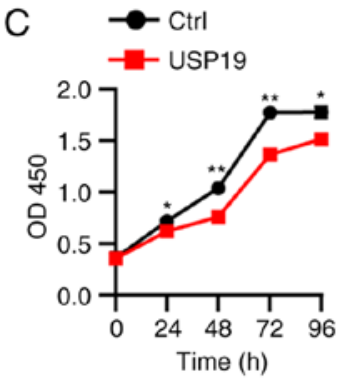

USP19
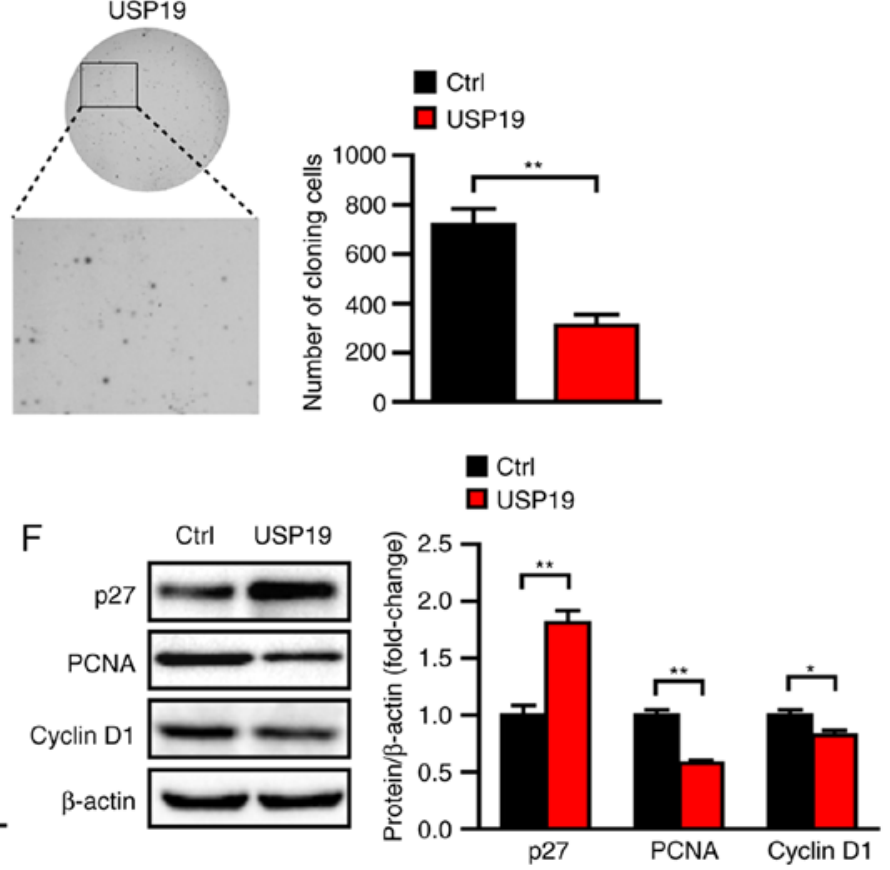

Figure 3. USP19 overexpression inhibits clear cell renal cell carcinoma cell proliferation in vitro. Expression of USP19 was evaluated by (A) RT-qPCR and (B) western blotting in USP19-overexpressing or control Caki-1 cells. (C) Cell Counting Kit-8 assays and (D) and soft agar assays were performed to detect cell proliferation in USP19-overexpressing or control Caki-1 cells. (E) RT-qPCR analysis of PCNA, cyclin D1 and p27 mRNA levels in USP19-overexpressing or control Caki-1 cells. (F) Western blot analysis of PCNA, cyclin D1 and p27 protein levels in USP19-overexpressing or control Caki-1 cells. $\beta$-actin was used as the loading control, and 3 replicates of the indicated proteins were quantified by Image Lab software. Data are presented as the mean \pm SD of three independent experiments. ${ }^{*} \mathrm{P}<0.05,{ }^{* *} \mathrm{P}<0.01$ vs. ctrl. USP, ubiquitin specific peptidase; RT-qPCR, reverse transcription-quantitative PCR; PCNA, proliferating cell nuclear antigen; OD, optical density. 


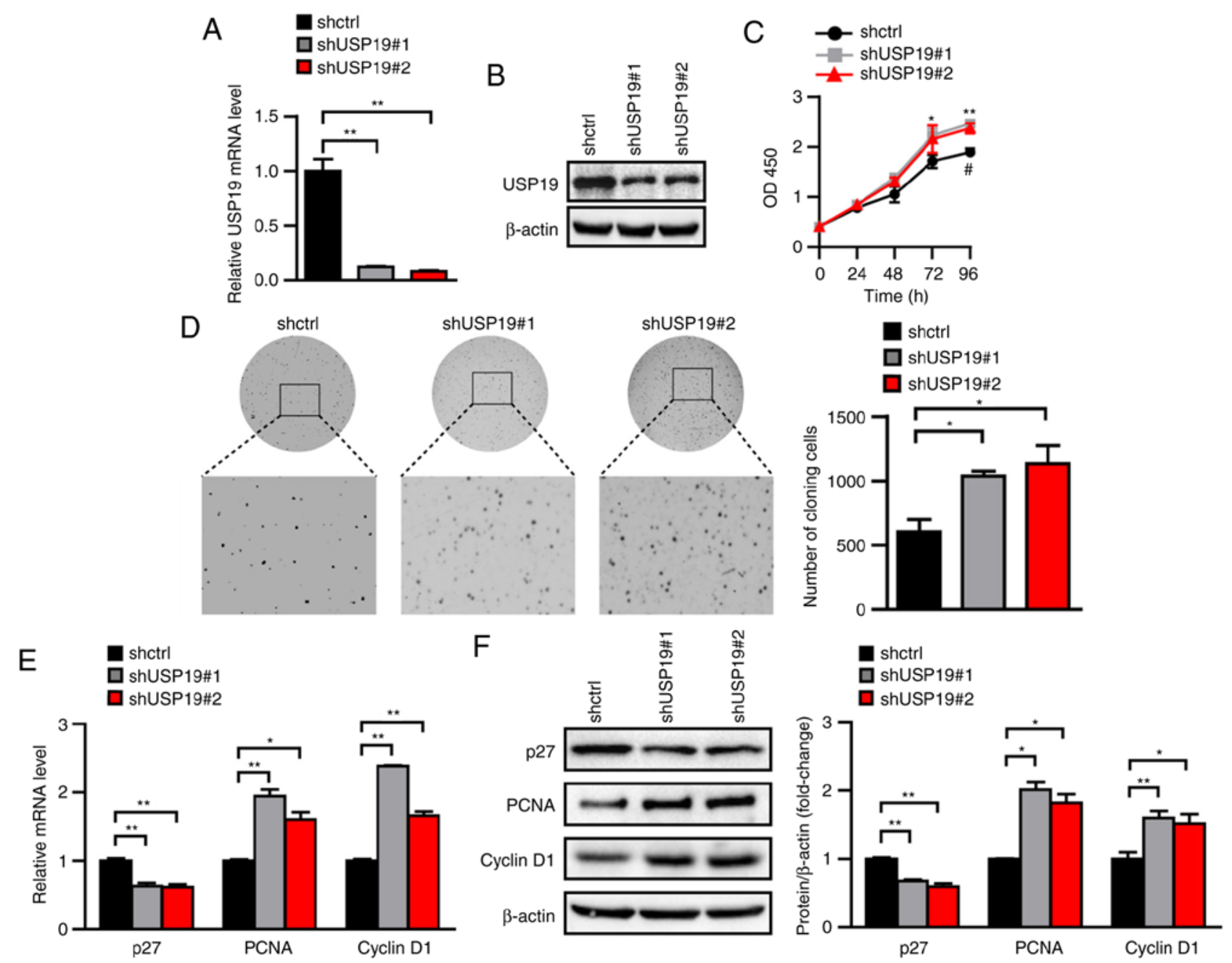

Figure 4. Knockdown of USP19 expression promotes clear cell renal cell carcinoma cell proliferation in vitro. Expression of USP19 was evaluated by (A) RT-qPCR and (B) western blotting in USP19 knockdown or control Caki-1 cells. (C) Cell Counting Kit-8 assays and (D) and soft agar assays were performed to detect cell proliferation in USP19 knockdown or control Caki-1 cells. (E) RT-qPCR analysis of PCNA, cyclin D1 and p27 mRNA levels in USP19 knockdown or control Caki-1 cells. (F) Western blot analysis of PCNA, cyclin D1 and p27 protein levels in USP19 knockdown or control Caki-1 cells. $\beta$-actin was used as the loading control, and 3 replicates of the indicated proteins were quantified by Image Lab software. Data are presented as the mean \pm SD of three independent experiments. ${ }^{*} \mathrm{P}<0.05,{ }^{* *} \mathrm{P}<0.01$ vs. shctrl; ${ }^{*} \mathrm{P}<0.05$ vs. shUSP19\#2. USP, ubiquitin specific peptidase; RT-qPCR, reverse transcription-quantitative PCR; PCNA, proliferating cell nuclear antigen; sh, short hairpin RNA; OD, optical density.

and 26 patients with ccRCC from GEO (GSE76207 and GSE102101) were employed. The results indicated that USP19 expression was significantly reduced in renal cancer tissues compared with in normal renal tissues $(\mathrm{P}<0.0001$; Fig. 1A-C). DNA CNVs are common in cancer and are responsible for the dysregulation of gene expression (20). To explain the mechanism of USP19 downregulation in ccRCC, the CNVs of the USP19 gene in 901 normal kidney tissue samples and 587 KIRC samples from TCGA were assessed. The results demonstrated that the USP19 gene copy number was significantly lower in ccRCC tissues than in normal tissues $\left(\mathrm{P}=2.2 \times 10^{-16}\right.$; Fig. 1D). These results indicated that USP19 expression is significantly decreased in ccRCC tissues, which may be due to copy number loss.

USP19 downregulation is associated with high tumor stage and poor prognosis. The associations between USP19 expression and clinicopathological features were further evaluated in TCGA KIRC cohort. Data showed that USP19 expression levels were significantly reduced in patients at a more advanced clinical stage $(\mathrm{P}<0.0001)$ and higher histological grade, $(\mathrm{P}=0.0086)$, but was not significant different between patients separated by age $(\mathrm{P}=0.2254)$ or sex $(\mathrm{P}=0.0933$; Table II).

Survival analysis revealed that patients with ccRCC with low USP19 expression exhibited significantly worse OS and DFS compared with those with high USP19 expression $(\mathrm{P}<0.05 ;$ Fig. $2 \mathrm{~A}$ and $\mathrm{B})$. In conclusion, these results suggested that USP19 downregulation is associated with high tumor stage and poor prognosis.

Overexpression of USP19 inhibits ccRCC cell proliferation in vitro. To explore the potential biological functions of USP19 in ccRCC, USP19-overexpressing cells were constructed by lentiviral infection of Caki-1 cells in vitro. USP19 mRNA and protein levels were significantly increased in USP19-overexpressing cells $(\mathrm{P}<0.05$; Fig. $3 \mathrm{~A}$ and $\mathrm{B})$. Caki-1 cell proliferation was assessed via CCK- 8 assays, and the results showed that proliferation was significantly 
A
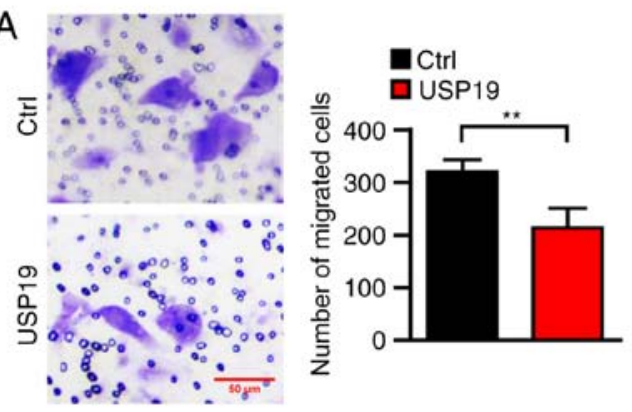

B

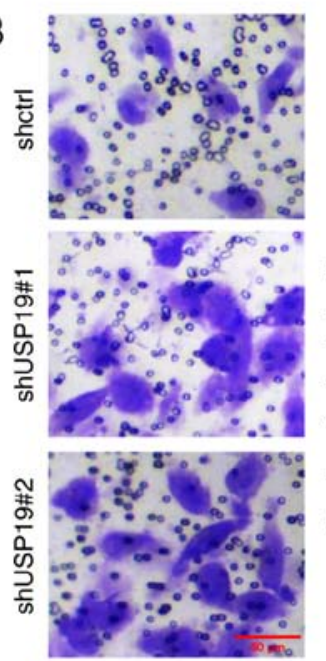

anctrl

口 shUSP19\#1

a shUSP19\#2

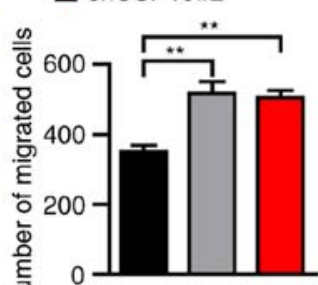

C

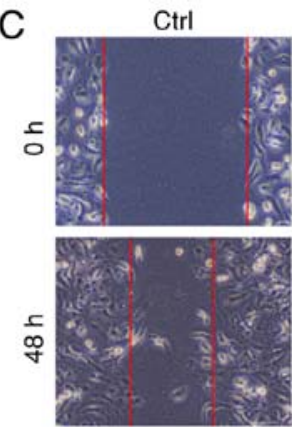

D
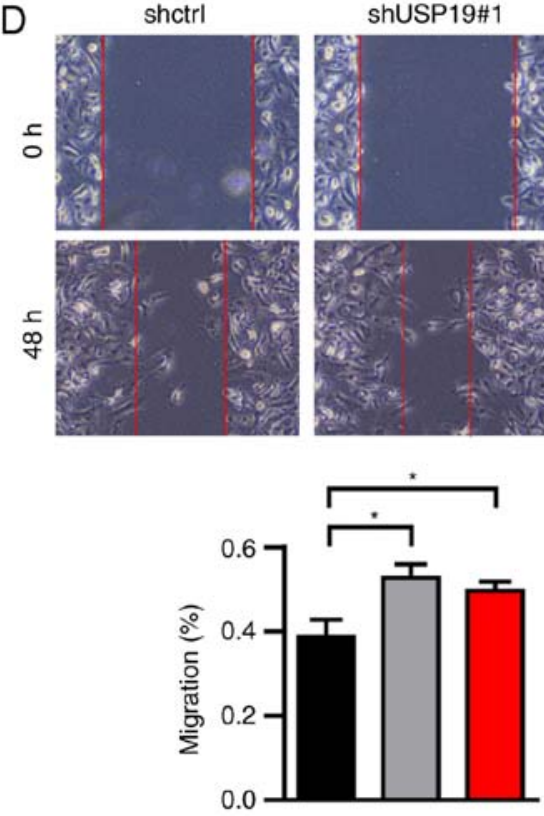

USP19

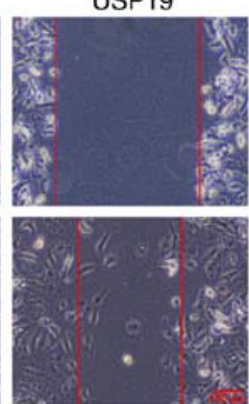

shUSP19\#1

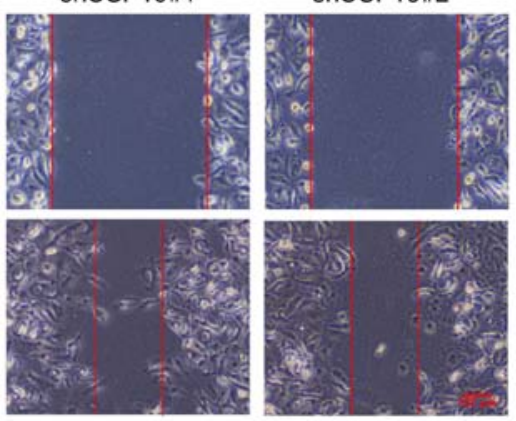

ahctrl

口 shUSP19\#1

口suSP19\#2
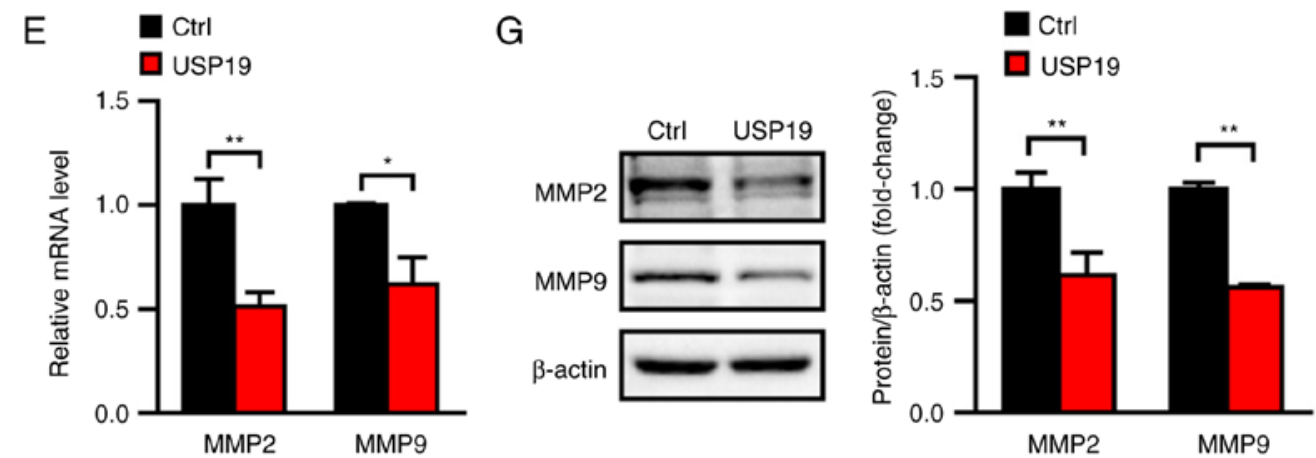

$\mathrm{F}$

a shctrl

口 ShUSP19\#1

口 shUSP19\#2

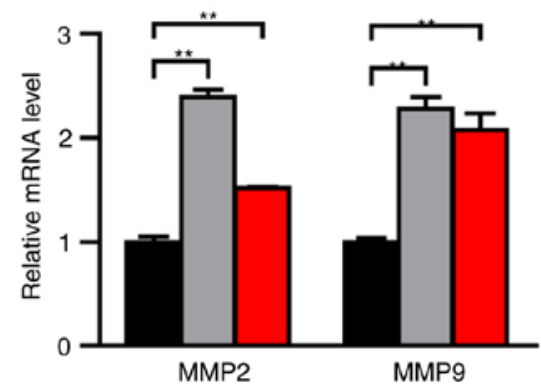

G

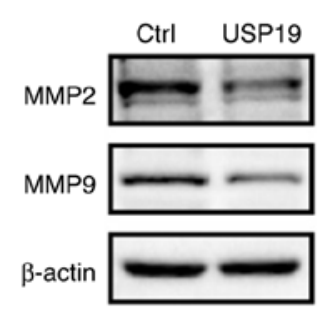

$\mathrm{H}$

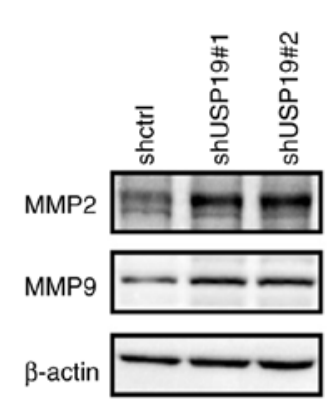

ashotrl

口 shUSP19\#1

口shUSP19\#2

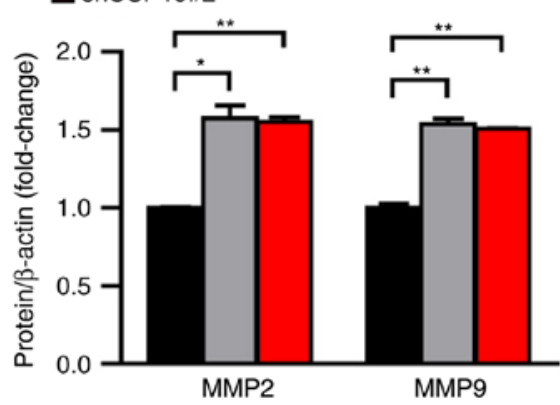

Figure 5. USP19 inhibits clear cell renal cell carcinoma cell migration in vitro. Migration abilities of (A) USP19-overexpressing and (B) knockdown Caki-1 cells were determined via Transwell assays; scale bar $=100 \mu \mathrm{m}, \mathrm{n}=4$. Migration abilities of (C) USP19-overexpressing and (D) knockdown Caki-1 cells were determined via wound healing assays; scale bar $=300 \mu \mathrm{m}, \mathrm{n}=4$. Reverse transcription-quantitative PCR analysis of MMP2 and MMP9 mRNA levels in (E) USP19-overexpressing or (F) knockdown Caki-1 cells; $n=3$. Protein expression of MMP2 and MMP9 in (G) USP19-overexpressing or (H) knockdown Caki-1 cells; $n=3$. Data are presented as the mean \pm SD. ${ }^{*} \mathrm{P}<0.05,{ }^{* *} \mathrm{P}<0.01$. USP, ubiquitin specific peptidase; RT-qPCR, reverse transcription-quantitative PCR; MMP, matrix metalloproteinase; sh, short hairpin RNA. 

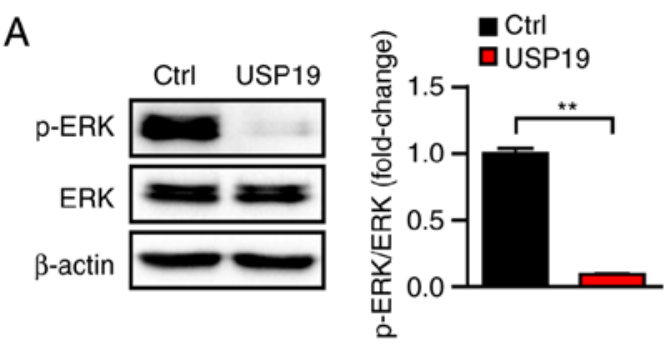

B
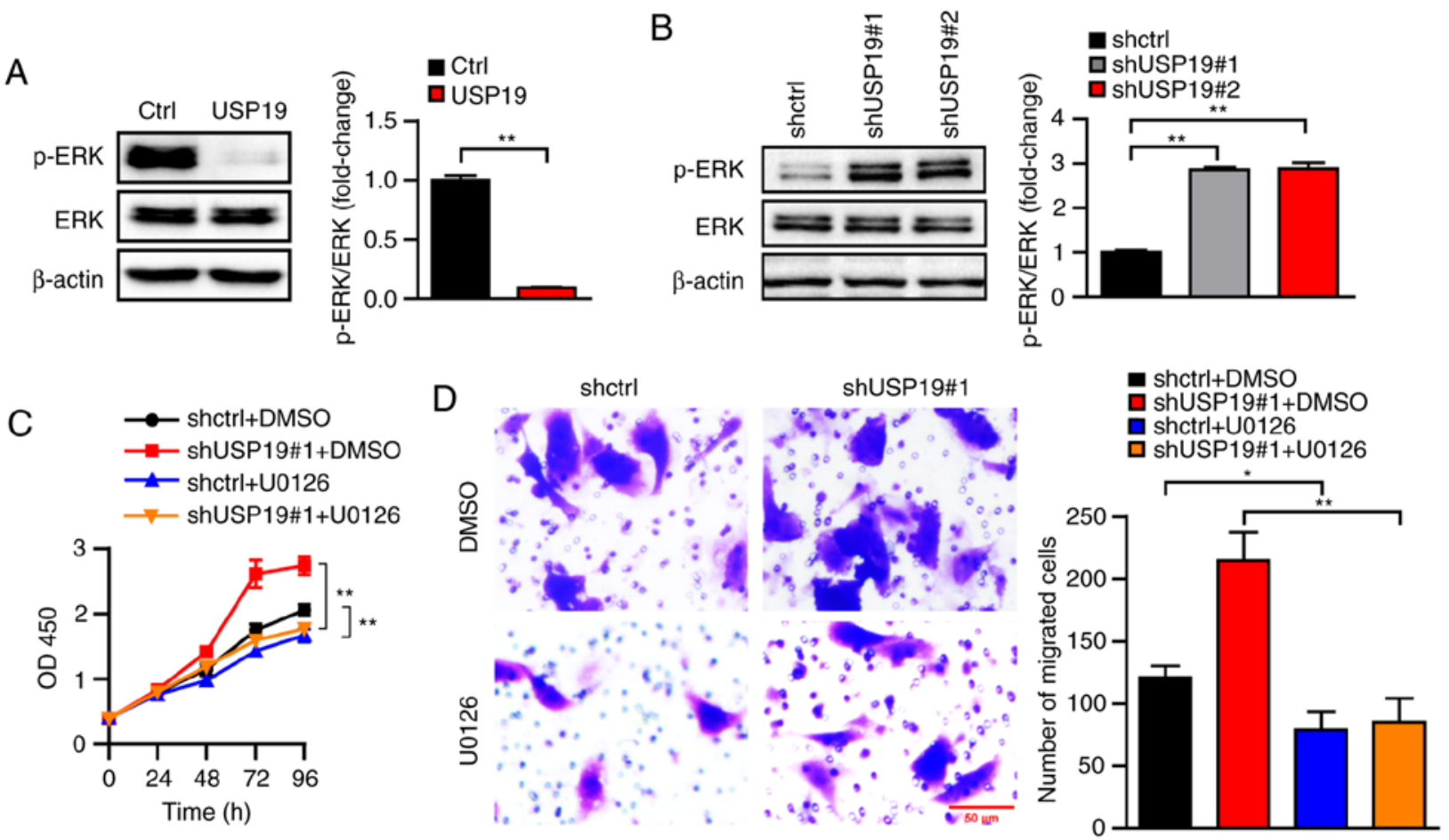

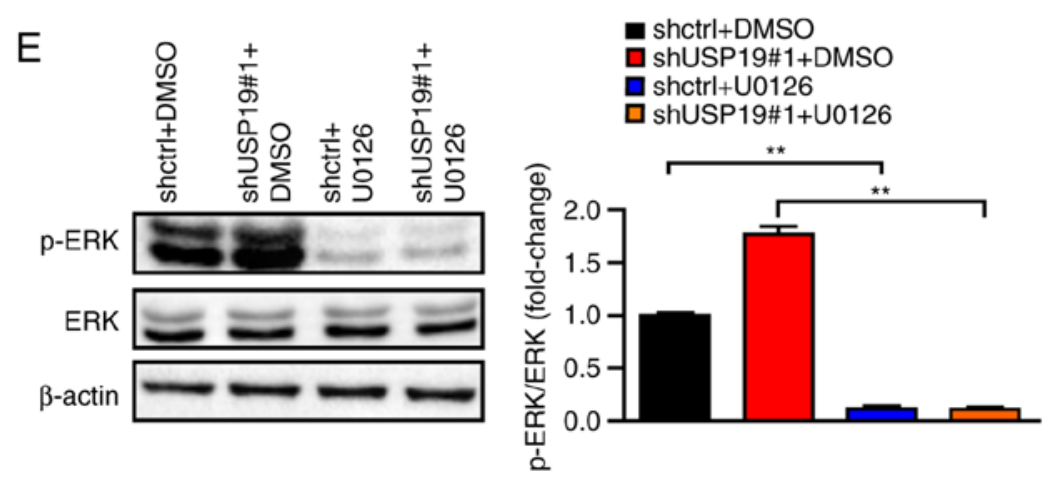

Figure 6. USP19 regulates clear cell renal cell carcinoma cell proliferation and migration by activating the ERK signaling pathway. (A) Levels of p-ERK and total ERK were measured by western blotting in USP19-overexpressing or control Caki-1 cells; $n=3$. (B) Levels of p-ERK and total ERK measured by western blotting in USP19 knockdown or control Caki-1 cells; $n=3$. (C) Cell Counting Kit-8 assays were performed to detect cell proliferation in USP19 knockdown or control Caki-1 cells treated with DMSO or U0126; $n=3$. (D) Transwell assays performed to detect cell migration in USP19 knockdown or control Caki-1 cells treated with DMSO or U0126; scale bar $=100 \mu \mathrm{m}, \mathrm{n}=4$. (E) Levels of p-ERK and total ERK in USP19 knockdown or control Caki-1 cells treated with DMSO or U0126; $n=3$. Data are presented as the mean $\pm \mathrm{SD}$. ${ }^{*} \mathrm{P}<0.05,{ }^{* *} \mathrm{P}<0.01$. USP, ubiquitin specific peptidase; $\mathrm{p}$, phosphorylated; sh, short hairpin RNA.

reduced in USP19-overexpressing Caki-1 cells compared with in control cells (all $\mathrm{P}<0.05$; Fig. 3C). Furthermore, soft agar assays showed that the colony number was significantly decreased for USP19-overexpressing Caki-1 cells compared with control cells $(\mathrm{P}<0.01$; Fig. 3D). Previous reports indicated that cyclin D1 and PCNA are involved in cell cycle control (21) and that p27 is a cyclin-dependent kinase inhibitor (22); these genes are thus markers of cell proliferation. Thus, mRNA levels of PCNA, cyclin D1, and p27 were evaluated via RT-qPCR. The results showed that mRNA levels of PCNA and cyclin D1 were downregulated when USP19 was overexpressed, whereas p27 was upregulated (all $\mathrm{P}<0.05$; Fig. 3E). Consistently, the protein levels of PCNA and cyclin D1 were decreased, and those of p27 were increased in USP19-ovexpressing cells compared with control cells (all $\mathrm{P}<0.05$; Fig. $3 \mathrm{~F}$ ). Taken together, the above results indicated that USP19 inhibits ccRCC cell proliferation.
Knockdown of USP19 expression promotes ccRCC cells proliferation in vitro. To examine whether USP19 knockdown promotes ccRCC cell proliferation, lentivirus-mediated shRNA constructs targeting different USP19 mRNA regions were applied to generate two stable USP19 knockdown cell lines. USP19 mRNA and protein levels were significantly reduced in stable USP19 knockdown Caki-1 cell lines compared with in shctrl Caki-1 cell lines (all $\mathrm{P}<0.05$; Fig. $4 \mathrm{~A}$ and $\mathrm{B}$ ). CCK- 8 and soft agar assays also showed that Caki-1 cell proliferation was increased following USP19 knockdown (all $\mathrm{P}<0.05$; Fig. 4C and D). Accordingly, the mRNA levels of PCNA and cyclin D1 were higher, and those of p27 were lower in the USP19 knockdown group compared with in the shctrl group (all $\mathrm{P}<0.05$; Fig. 4E). Similarly, the protein levels of PCNA and cyclin D1 were increased, while those of p27 were decreased by USP19 knockdown (all P<0.05; Fig. 4F). 


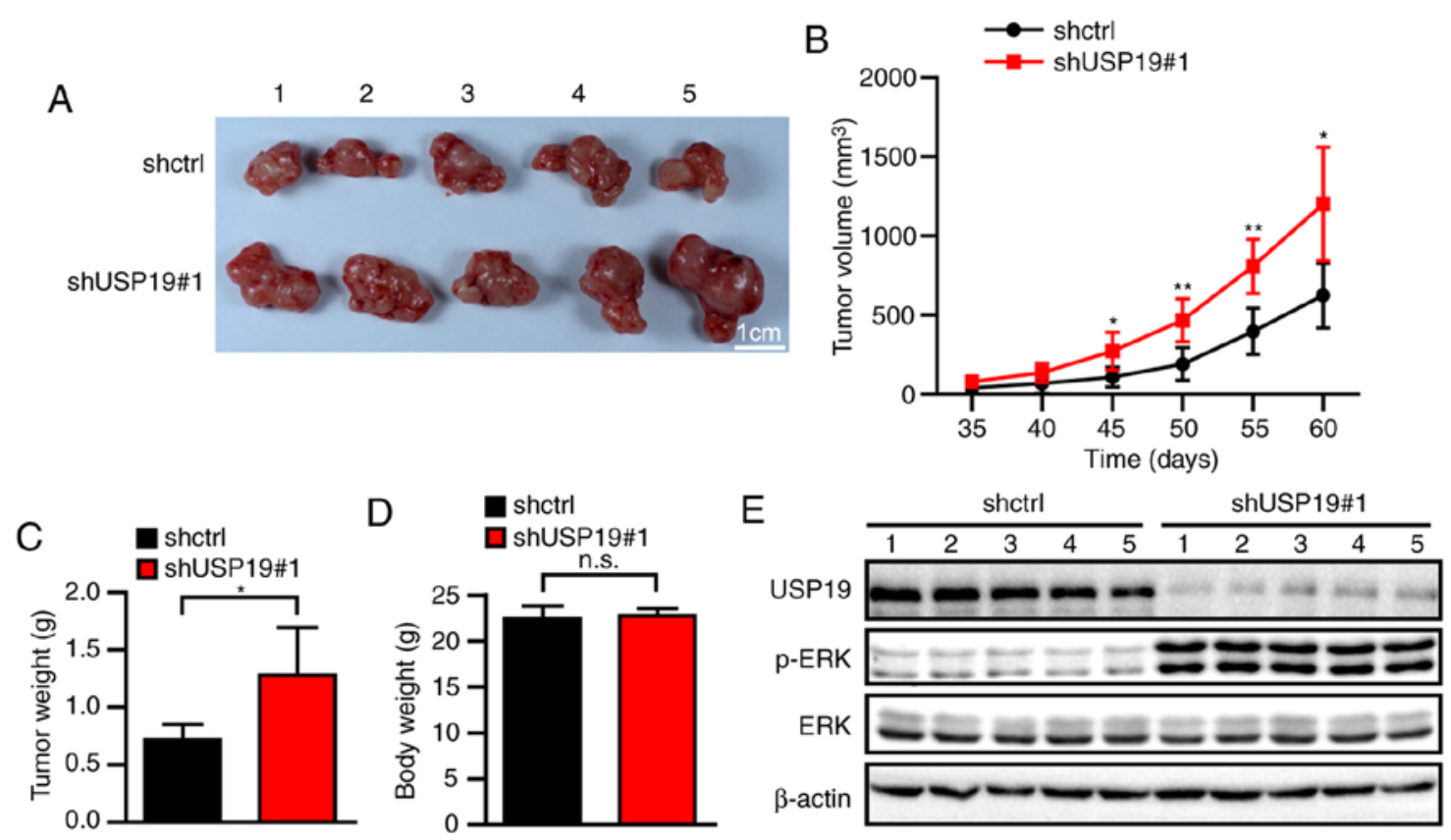

Figure 7. USP19 inhibits the tumorigenesis of clear cell renal cell carcinoma cells in vivo. (A) Photographs of mouse xenografts generated by subcutaneous injection of Caki-1 cells; $n=5 /$ group. (B) Tumor volumes were evaluated after injection every 5 days for 60 days. (C) Weights of the xenografts were measured 60 days after injection. (D) Body weights of the mice were measured 60 days after injection. (E) Protein levels of USP19, p-ERK and total ERK in tumor tissue were analyzed via western blotting. Data are presented as the mean $\pm \mathrm{SD}$. ${ }^{*} \mathrm{P}<0.05,{ }^{* * *} \mathrm{P}<0.01$ vs. shctrl. USP, ubiquitin specific peptidase; $\mathrm{p}$, phosphorylated; sh, short hairpin RNA; n.s., not significant.

These results indicated that USP19 knockdown promotes ccRCC cell proliferation.

USP19 inhibits ccRCC cell migration in vitro. Having observed a significant change in cell proliferation following the dysregulation of USP19 expression, the effects of USP19 on cell migration were further evaluated using Transwell and wound healing assays. Transwell assays showed that cell migration was impaired in USP19-overexpressing Caki-1 cells, whereas it was promoted in USP19 knockdown Caki-1 cells compared with the respective negative controls (all $\mathrm{P}<0.01$; Fig. 5A and B). Similarly, microscopic examination of wounds at 0 and $48 \mathrm{~h}$ showed that the migration rate in the USP19 overexpression group was significantly reduced, whereas it was increased in USP19 knockdown group compared with the control groups (all $\mathrm{P}<0.05$; Fig. $5 \mathrm{C}$ and $\mathrm{D}$ ). MMP2 and MMP9 are frequently with the malignant phenotype of tumor cells, and are commonly used as malignant tumor migration markers $(23,24)$. RT-qPCR and western blot data showed that the expression levels of MMP2 and MMP9 were lower in USP19-overexpressing cells and higher in USP19-knockdown cells than in control cells (all $\mathrm{P}<0.05$; Fig. 5E-H). These data indicated that USP19 inhibits ccRCC cell migration in vitro.

USP19 regulates ccRCC cell proliferation and migration by regulating the ERK signaling pathway. ERK/mitogen-activated protein kinase (MAPK) signaling has been shown to be involved in various cancer-associated pathological processes $(25,26)$. To investigate the underlying molecular signaling pathways that participate in USP19-mediated inhibition of proliferation and migration in ccRCC cancer cells, ERK activity was determined in USP19-overexpressing and knockdown Caki-1 cells. Of note, it was found that total ERK protein levels were not notably changed in the USP19 knockdown or overexpression ccRCC cells. However, USP19 overexpression clearly reduced p-ERK levels, while USP19 knockdown increased ERK phosphorylation (all $\mathrm{P}<0.01$; Fig. 6A and B), indicating that ERK may mediate the functions of USP19 in ccRCC cancer cells. Thus, the effects of the ERK inhibitor U0126 on the functions of USP19 in ccRCC cancer cells were determined. As the CCK-8 assay showed, U0126 significantly rescued the increased Caki-1 cell proliferation induced by USP19 knockdown (all $\mathrm{P}<0.01$; Fig. 6C). Transwell assays also indicated that the promoting effects of USP19 knockdown on Caki-1 cell migration were reversed by U0126 (all P<0.05; Fig. 6D). Treatment of U0126 led to a significant decrease in p-ERK levels, whilst not notably affecting the total ERK protein levels (all $\mathrm{P}<0.01$; Fig. $6 \mathrm{E}$ ). Hence, these results demonstrated that USP19 regulates ccRCC proliferation and migration through its regulation of ERK signaling.

USP19 inhibits tumorigenesis in ccRCC cells in vivo. To investigate the effect of USP19 in vivo, nude mice were inoculated with shctrl- or shUSP19\#1-infected Caki-1 cells. Compared with the tumors created by the shctrl cells, the tumors derived from the shUSP19\#1-infected Caki-1 cells were significantly larger (all $\mathrm{P}<0.05$; Fig. 7A and B). Consistently, the tumor weights at 60 days were significantly heavier in the shUSP19\#1 group than the shctrl group $(\mathrm{P}<0.05$; Fig. $7 \mathrm{C})$, whereas the body weights at 60 days of the mice in the two groups were not significantly different ( $P>0.05$; Fig. 7D) Then, western blot experiments revealed notably increased p-ERK levels in the tumor tissues derived from the shUSP19\#1 group compared with those derived from the shctrl group (Fig. 7E). These find- 
ings indicated that USP19 downregulation promotes ccRCC tumor growth in vivo.

\section{Discussion}

The present study revealed that USP19 may function as a tumor suppressor in ccRCC, and that USP19 expression is significantly reduced in ccRCC tissues, based on multiple downloaded datasets. Furthermore, the present study also demonstrated that USP19 downregulation in ccRCC was associated with more advanced tumors and predicted unfavorable prognostic outcomes in a TCGA cohort.

CNV (20), DNA methylation aberration (27) and transcriptional dysregulation (28) are all important events for dysregulation of gene expression during various diseases. In cancer cells, in which somatic mutations are easily induced, CNVs play an important role in the somatic mutation-induced dysregulation of cancer genes (29-31). In the present study, by analyzing CNV data from TCGA database, it was revealed that the copy number of USP19 was notably reduced in ccRCC tissues, suggesting that USP19 loss may be due to copy number loss, which requires further investigation.

CCK-8 assays are conducted to assess cancer cell proliferation abilities, and Transwell and wound healing assays are used to evaluate migration abilities (32-35). To investigate the observations from the bioinformatics analyses, in vitro cell line experiments were conducted, indicated that USP19 overexpression suppressed proliferation and migration, whereas USP19 knockdown promoted proliferation and migration. At the molecular level, USP19 overexpression decreased the expression of the proliferation-promoting genes PCNA and cyclin D1, and increased the expression of the proliferation-inhibiting gene p27; conversely, USP19 overexpression decreased the expression of the migration-associated genes MMP2 and MMP9. Conversely, depletion of USP19 inhibited proliferation in DU145, PC-3 and 22RV1 prostate cancer cells, indicating that USP19 has distinct functions in different tumors (36).

Aberrant ERK-MAPK pathway signaling contributes to cell proliferation and migration in various types of cancer $(37,38)$. In the present study, USP19 overexpression reduced p-ERK levels. Moreover, USP19 knockdown promoted ERK phosphorylation, as well as promoting ccRCC proliferation in vitro and in vivo. Therefore, these results suggested that the molecular mechanism underlying USP19-mediated proliferation and migration inhibition in ccRCC may depend on inactivation of the ERK-MAPK signaling pathway. However, one limitation of the present study is that the colocalization of USP19 and p-ERK was not confirmed in mouse tumor tissues, due to low quantities and poor specificity of the USP19 antibody.

$\mathrm{Wu}$ et al (39) previously reported that USP19 expression was lower in ccRCC tissue compared with in normal kidney tissue by analyzing TCGA KIRC data. Liu et al (40) analyzed gene and isoform expression signatures associated with tumor stage in KIRC and found uc003cvz.3, the major isoform of USP19, was significantly downregulated in patients with stage IV KIRC, whereas higher uc003cvz.3 expression suggested improved survival rates. However, these two studies only showed the clinical relationship of USP19 and ccRCC by analyzing databases; they did not investigate the biological functions of USP19 in ccRCC cells. Conversely, the present study demonstrated that USP19 overexpression inhibited ccRCC cell proliferation and migration in vitro; conversely, USP19 knockdown promoted proliferation and migration in vitro, as well as promoting tumor growth in vivo. To the best of our knowledge, this is the first study to investigate the biological functions and molecular mechanisms of USP19 in ccRCC. These findings expand current knowledge regarding USP19, and suggest that USP19 may play an important role in the development and progression of ccRCC.

\section{Acknowledgments}

Not applicable.

\section{Funding}

This work was supported by Hubei Health Research Foundation (grant no. WJ2019F039).

\section{Availability of data and materials}

The datasets used and/or analyzed during the present study are available from the corresponding author on reasonable request.

\section{Authors' contributions}

ZT and JZ designed the study. WH, YS, XF, XW and GZ performed the experiments. WH, YS, CS, TD, TY and GW performed data analysis. WH, YS, ZT and JZ contributed to manuscript revisions and all the authors reviewed the manuscript. All authors read and approved the final manuscript.

\section{Ethics approval and consent to participate}

All protocols involving animals were approved by the Institutional Animal Care and Use Committee of the Institute of Model Animals of Wuhan University.

\section{Patient consent for publication}

Not applicable.

\section{Competing interests}

The authors declare that they have no competing interests.

\section{References}

1. Bray F, Ferlay J, Soerjomataram I, Siegel RL, Torre LA and Jemal A: Global cancer statistics 2018: GLOBOCAN estimates of incidence and mortality worldwide for 36 cancers in 185 countries. CA Cancer J Clin 68: 394-424, 2018.

2. Rini BI, Campbell SC and Escudier B: Renal cell carcinoma. Lancet 373: 1119-1132, 2009.

3. Delahunt B, Bethwaite PB and Nacey JN: Outcome prediction for renal cell carcinoma: Evaluation of prognostic factors for tumors divided according to histological subtype. Pathology 39: 459-465, 2007.

4. Lin YF, Chou JL, Chang JS, Chiu IJ, Chiu HW and Lin YF: Dysregulation of the miR-25-IMPA2 axis promotes metastatic progression in clear cell renal cell carcinoma. EBioMedicine 45: 220-230, 2019. 
5. Afriansyah A, Hamid AR, Mochtar CA and Umbas R: Targeted therapy for metastatic renal cell carcinoma. Acta Med Indones 48: 335-347, 2016

6. Husnjak K and Dikic I: Ubiquitin-binding proteins: Decoders of ubiquitin-mediated cellular functions. Annu Rev Biochem 81: 291-322, 2012

7. Harada K, Kato M and Nakamura N: USP19-mediated deubiquitination facilitates the stabilization of HRD1 ubiquitin ligase. Int J Mol Sci 17: pii: E1829, 2016.

8. Fraile JM, Quesada V, Rodríguez D, Freije JM and López-Otín C: Deubiquitinases in cancer: New functions and therapeutic options. Oncogene 31: 2373-2388, 2012.

9. Hu M, Li P, Li M, Li W, Yao T, Wu JW, Gu W, Cohen RE and Shi Y: Crystal structure of a UBP-family deubiquitinating enzyme in isolation and in complex with ubiquitin aldehyde. Cell 111: 1041-1054, 2002.

10. Hassink GC, Zhao B, Sompallae R, Altun M, Gastaldello S, Zinin NV, Masucci MG and Lindsten K: The ER-resident ubiquitin-specific protease 19 participates in the UPR and rescues ERAD substrates. EMBO Rep 10: 755-761, 2009.

11. Jin S, Tian S, Chen Y, Zhang C, Xie W, Xia X, Cui J and Wang RF USP19 modulates autophagy and antiviral immune responses by deubiquitinating Beclin-1. EMBO J 35: 866-880, 2016.

12. Gierisch ME, Pedot G, Walser F, Lopez-Garcia LA, Jaaks P, Niggli FK and Schäfer BW: USP19 deubiquitinates EWS-FLI1 to regulate Ewing sarcoma growth. Sci Rep 9: 951, 2019.

13. Shahriyari L, Abdel-Rahman M and Cebulla C: BAP1 expression is prognostic in breast and uveal melanoma but not colon cancer and is highly positively correlated with RBM15B and USP19. PLoS One 14: e0211507, 2019.

14. Eikrem O, Beisland C, Hjelle K, Flatberg A, Scherer A, Landolt L, Skogstrand T, Leh S, Beisvag V and Marti HP: Transcriptome sequencing (RNAseq) enables utilization of Formalin-fixed, paraffin-embedded biopsies with clear cell renal cell carcinoma for exploration of disease biology and biomarker development. PLoS One 11: e0149743, 2016.

15. Yao X, Tan J, Lim KJ, Koh J, Ooi WF, Li Z, Huang D, Xing M, Chan YS, Qu JZ, et al: VHL deficiency drives enhancer activation of oncogenes in clear cell renal cell carcinoma. Cancer Discov 7: 1284-1305, 2017

16. R Core Team: R: A language and environment for statistical computing. R Foundation for Statistical Computing, Vienna, 2104

17. Love MI, Huber W and Anders S: Moderated estimation of fold change and dispersion for RNA-seq data with DESeq2. Genome Biol 15: 550, 2014

18. Livak KJ and Schmittgen TD: Analysis of relative gene expression data using real-time quantitative PCR and the 2(-Delta Delta C(T)) method. Methods 25: 402-408, 2001.

19. National Research Council (US) Committee for the Update of the Guide for the Care and Use of Laboratory Animals: Guide for the Care and Use of Laboratory Animals, 8th edition. National Academies Press (US), Washington, DC, 2011.

20. Macè A, Kutalik Z and Valsesia A: Copy number variation. Methods Mol Biol 1793: 231-258, 2018.

21. Zińczuk J, Zareba K, Guzińska-Ustymowicz K1, Kedra B Kemona A and Pryczynicz A: Expression of chosen cell cycle and proliferation markers in pancreatic intraepithelial neoplasia. Prz Gastroenterol 13: 118-126, 2018.

22. Sarsik B, Doganavsargil B, Simsir A, Yazici A, Pehlivanoglu B, $\mathrm{Cal} C$ and Sen S: P21 and p27 immunoexpression in upper urinary tract urothelial carcinomas. Pathol Oncol Res 22: 839-845, 2016

23. Egeblad M and Werb Z: New functions for the matrix metalloproteinases in cancer progression. Nat Rev Cancer 2: 161-174, 2002.

24. Björklund $\mathrm{M}$ and Koivunen E: Gelatinase-mediated migration and invasion of cancer cells. Biochim Biophys Acta 1755: 37-69, 2005 .
25. Wang D, Wang D, Wang N, Long Z and Ren X: Long Non-coding RNA BANCR promotes endometrial cancer cell proliferation and invasion by regulating MMP2 and MMP1 via ERK/MAPK signaling pathway. Cell Physiol Biochem 40 644-656, 2016.

26. Tian XQ, Guo FF, Sun DF, Wang YC, Yang L, Chen SL, Hong J and Fang JY: Downregulation of ZNF278 arrests the cell cycle and decreases the proliferation of colorectal cancer cells via inhibition of the ERK/MAPK pathway. Oncol Rep 38: 3685-3692, 2017.

27. He W, Ju D, Jie Z, Zhang A, Xing X and Yang Q: Aberrant $\mathrm{CpG}$-methylation affects genes expression predicting survival in lung adenocarcinoma. Cancer Med 7: 5716-5726 2018.

28. Schuijers J, Manteiga JC, Weintraub AS, Day DS, Zamudio AV, Hnisz D, Lee TI and Young RA: Transcriptional dysregulation of MYC reveals common enhancer-docking mechanism. Cell Rep 23: 349-360, 2018.

29. Gu X, Coates PJ, Boldrup L, Wang L, Krejci A, Hupp T, Fahraeus R, Norberg-Spaak L, Sgaramella N, Wilms T and Nylander K: Copy number variation: A prognostic marker for young patients with squamous cell carcinoma of the oral tongue. J Oral Pathol Med 48: 24-30, 2019.

30. Wee Y, Wang T, Liu Y, Li X and Zhao M: A pan-cancer study of copy number gain and up-regulation in human oncogenes. Life Sci 211: 206-214, 2018.

31. Wang L, Zhao H, Xu Y, Li J, Deng C, Deng Y, Bai J, Li X, Xiao Y and Zhang Y: Systematic identification of lincRNA-based prognostic biomarkers by integrating lincRNA expression and copy number variation in lung adenocarcinoma. Int $\mathrm{J}$ Cancer 144: 1723-1734, 2019

32. Duan X, Jiang B, Yang J, Zhou L, Tian B and Mao X: FOXP3 inhibits MYC expression via regulating miR-198 and influences cell viability, proliferation and cell apoptosis in HepG2. Cancer Med 7: 6182-6192, 2018

33. He J, Yu L, Wang CM and Zhou XF: MiR-1275 promotes non-small cell lung cancer cell proliferation and metastasis by regulating LZTS3 expression. Eur Rev Med Pharmacol Sci 22: 2680-2687, 2018.

34. Chen Y, Zhang L, Liu L, Sun S, Zhao X, Wang Y, Zhang Y, Du J and Gu L: Rasip1 is a RUNX1 target gene and promotes migration of NSCLC cells. Cancer Manag Res 10: 4537-4552, 2018.

35. Wang Y, Zheng C, Li T, Zhang R, Wang Y, Zhang J, He Q, Sun $Z$ and Wang $X$ : Long noncoding RNA Z38 promotes cell proliferation and metastasis and inhibits cell apoptosis in human gastric cancer. Oncol Lett 16: 6051-6058, 2018.

36. Lu Y, Bedard N, Chevalier S and Wing SS: Identification of distinctive patterns of USP19-mediated growth regulation in normal and malignant cells. PLoS One 6: e15936, 2011.

37. Bao H, Guo CG, Qiu PC, Zhang XL, Dong Q and Wang YK: Long non-coding RNA Igf2as controls hepatocellular carcinoma progression through the ERK/MAPK signaling pathway. Oncol Lett 14: 2831-2837, 2017.

38. Li B, Liu YH, Sun AG, Huan LC, Li HD and Liu DM: MiR-130b functions as a tumor promoter in glioma via regulation of ERK/MAPK pathway. Eur Rev Med Pharmacol Sci 21: 2840-2846, 2017.

39. Wu M, Tu HQ, Chang Y, Tan B, Wang G, Zhou J, Wang L Mu R and Zhang WN: USP19 deubiquitinates HDAC1/2 to regulate DNA damage repair and control chromosomal stability. Oncotarget 8: 2197-2208, 2017.

40. Liu Q, Zhao S, Su PF and Yu S: Gene and isoform expression signatures associated with tumor stage in kidney renal clear cell carcinoma. BMC Syst Biol 7 (Suppl 5): S7, 2013.

This work is licensed under a Creative Commons Attribution-NonCommercial-NoDerivatives 4.0 International (CC BY-NC-ND 4.0) License. 